\title{
Control System Design Based on a Universal First Order Model with Time Delays
}

\author{
T. Vyhlídal, P. Zítek
}

\begin{abstract}
An original modelling approach for SISO systems is presented, based on a first order model with more than one delay in its structure. By means of this model it is possible truly to hit off the properties of systems which are conventionally described by higher order models. The identification method making use of a relay feedback test combined with transient responses of the system has proved to be suitable for assessing the model parameters. With respect to its plain structure the model is well suited to be applied in the framework of an internal model control scheme (IMC). The resultant control algorithm with only one optional parameter is very simple and can easily be implemented, for example by means of a programmable controller (PLC).
\end{abstract}

Keywords: time delay system, internal model control, system eigenvalues, control parameter setting.

\section{Introduction}

Control problems of time delay systems have been solved since the very beginning of modern control theory. The well-known idea of compensating input delay by means of a special control loop arrangement was introduced by Smith [1]. In fact the idea of the single input delay in the plant model is also considered in the Ziegler and Nichols method of controller setting. Series linkage of the delay and other parts of the model is often used in control engineering for describing of systems with a significant dead time. A conventionally applied model for such systems is assumed with the following transfer function

$$
G(s)=\frac{K \exp (-s \tau)}{(T s+1)^{n}}
$$

where $n=1,2, \ldots$ is the order of the model, $K$ is steady state gain, $T$ is time constant and $t$ is input time delay. The first order model $n=1$ is often used in practice. Three parameters $K, T$ and $\tau$ can be estimated for example from the step response of the system (see Figure 3 ). It should be noted that the model obtained in this way often truly describes only the transient behaviour of the system. This results from the fact that most real systems have more complicated dynamic behaviour, which can be only very roughly described by the first order model with only input time delay. Discrepancies between the transient and frequency responses of the model and the real system often occur if the first order model is used. The use of a higher order model $(n>1)$ can reduce these discrepancies, but it causes increasing complexity of the model structure. On the other hand, the first order model is advantageous if the model-based control strategy is to be applied. The low order of the model brings about the low order of the final controller model desirable for its implementation.

\section{First order model with time delays}

Instead of the standard approximation (1) let us assume an alternative model of the system

$$
G(s)=\frac{K \exp (-s \tau)}{T s+\exp (-s \phi)}
$$

corresponding to the delayed differential equation

$$
T y^{\prime}(t)+y(t-\phi)=K u(t-\tau)
$$

where $u$ and $y$ are system input and system output respectively, $t$ is time, $K$ is steady state gain, $T$ is time constant, $\tau$ is input time delay and $\phi$ is state delay. In effect, state delay $\phi$ plays an analogous role as the $n$-th power in the denominator of (1). In combination with the input delay it allows (1) dynamics to be described by means of model (2). For the sake of model (2) generalisation it is useful to introduce a reference model with the following relative parameters: $K=1, T=1, \phi^{*}=\phi / T, \tau^{*}=\tau / T$ and $t^{*}=t / T$. The dynamic properties of the linear model can be determined by the solutions of its characteristic equation, i.e. the system poles. The structure of the characteristic equation of model (2) is very simple

$$
M(s)=s+\exp \left(-s \phi^{*}\right)=0
$$

but it is not easy to find a sufficient set of solutions. Because of the presence of the exponential term, equation (4) is not algebraic but transcendental and therefore it has infinitely many poles. On the other hand, only a few of this set of poles have a significant influence on the system behaviour [2]. The distance between the appropriate pole and the origin of complex plane determines the pole significance. The more distant the pole, the less significant. Poles may be either real or complex conjugate. Real poles represent damped modes, while complex poles $s=\beta \pm$ j $\omega$ represent oscillatory modes of the system dynamics. The ratio between the real and imaginary part of a complex pole is known as relative damping $\rho=|\beta| / \omega$.

The location of the poles is dependent on the model parameters. The trajectories of the poles with respect to the value of state delay $\phi^{*}$ are depicted in Figure 1 . As can be seen, if $\phi^{*}=0$ system (2) has only one real pole in the selected region. This real pole denoted as s1 moves to the left with increasing value of $\phi^{*}$. It should be recalled that since $\phi^{*} \neq 0$ equation (4) is transcendental, with infinitely many solutions. Another real pole emerges in the left part of Figure 1 and it moves to the right as $\phi^{*}$ increases. The case of double pole $s_{1,2}$ occurs with $\phi^{*}=\exp (-1)$. It is the highest value of $\phi^{*}$ for which equation (4) has real solutions. For higher $\phi^{*}$ the poles 
are complex conjugate only. It is apparent from Figure 1 that the couple $s_{1,2}$ is always the nearest one to the origin of the complex plane. This determines its dominant influence in the system dynamics. Common intersection points of all the pole trajectories are $\operatorname{Im}(M(s))= \pm 1$ and $\operatorname{Re}(M(s))=0$ (Marshal et al [3]). The dominant couple reaches this point for $\phi^{*}=\pi / 2$. This is the ultimate value for which model (2) is stable. For values of $\phi^{*}>\pi / 2$, the dominant couple of poles enters the right half of the complex plane, which indicates loss of stability.

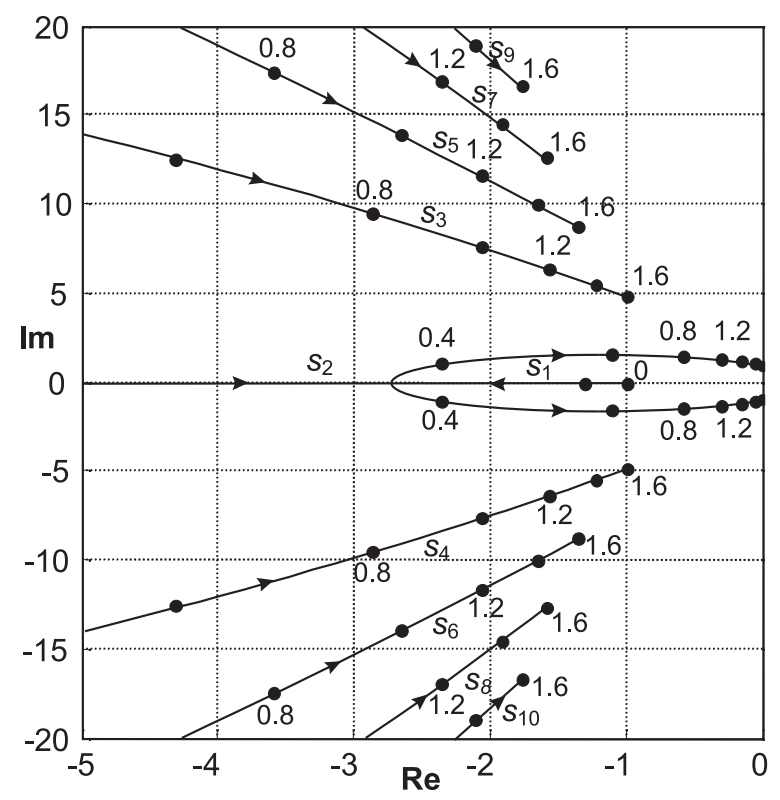

Fig. 1: Pole trajectories with respect to $\phi^{*} \in\langle 0 ; \pi / 2\rangle$

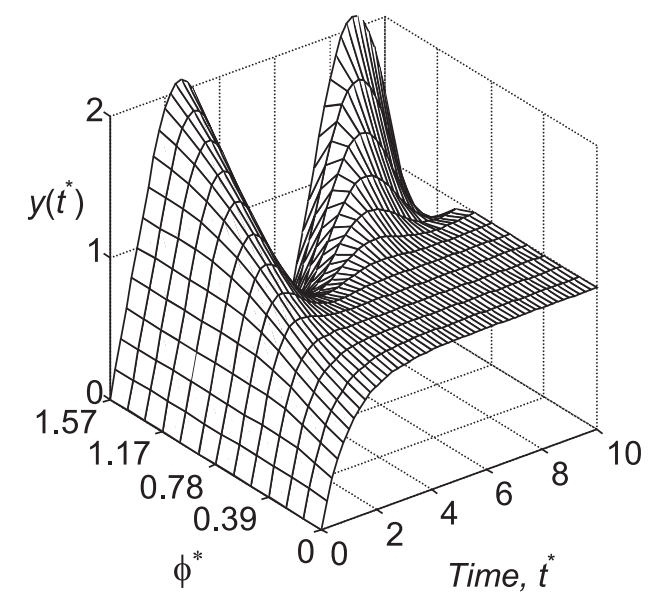

Fig. 2: Step responses of model (2) with respect to $\phi^{*} \in\langle 0 ; \pi / 2\rangle$

The significance of $\phi^{*}$ is also demonstrated in the step responses of model (2) (see Figure 2). The relationship between the position of dominant poles $s_{1,2}$ and appropriate responses is apparent. The smaller the value of the relative damping $\rho=|\beta| / \omega$ of the dominant poles, the more oscillatory the step response.

There is a class of systems for which not only distribution of poles but also distribution of zeros is important. These sys- tems cannot be truly describesd by models (1) or (2). The general differential equation with derivations on both sides is usually used for describing such systems. Zeros can be added to the first order model (2) by means of enlargement of the numerator by the term $P s+\exp (-s \psi)$.

$$
G(s)=\frac{[P s+\exp (-s \psi)] K \exp (-s \tau)}{T s+\exp (-s \phi)} .
$$

Time constant $P$ and time delay $\psi$ play an analogous role in the numerator to parameters $T$ and $\phi$ in the denominator. The ratio $\psi^{*}=\psi / P$ determines the system zeros distribution, in accordance with Figure 1.

\section{Identification of the system parameters}

The step response is often used for a first estimation of system properties. It is customary and a relatively easy matter to assess the input time delay, time constant and steady state gain. These parameters are sufficient for the system representation by means of model (1) if $n=1$. The steady state gain is simply given by $K=\Delta \mathrm{y} / \Delta \mathrm{u}$. The tangent in the inflexion point of the step response assesses parameters $T$ and $\tau$. As is shown in Figure 3 these parameters can be read from the time axis of the step response.

If the model with two delays (2) is used, its parameters can also be estimated from the step response. The tangent in the inflection point of the step response also assesses $T$ and $\tau$. The remaining parameter $\phi$ influences the position of the inflection point of the step response. It is usually a relatively easy task to find the tangent in the inflexion point, but it is rather complicated to find the exact position of this point, especially if the response is influenced by the measurement or the system noise.

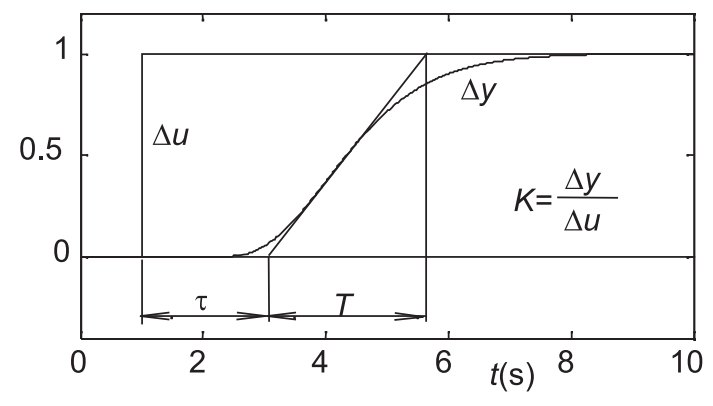

Fig. 3: Step response

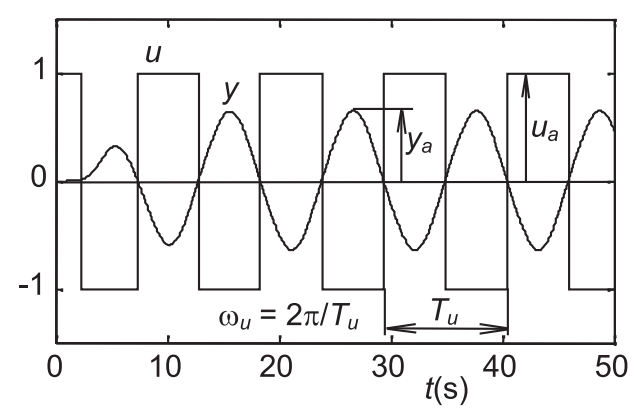

Fig. 4: Oscillating motion of relay feedback 
An identification method based on the relay feedback test (Astrom, Hagglund [4]) is suggested in order to find the value of parameter $\phi$. The relay is usually used for providing on-off control of the system. For a large class of systems, relay feedback gives an oscillating motion (see Figure 4), the frequency of which is close to the system ultimate frequency $\omega_{u}$. The system ultimate gain is approximately given by Astrom and Hagglund as

$$
k_{u}=\frac{4 u_{a}}{\pi y_{a}}
$$

where $u_{a}$ a $y_{a}$ are the amplitudes of the relay and system output, respectively. The point of the system frequency response with argument $\Phi(\omega)=-\pi$ is described by the above ultimate parameters. On the basis of this point two parameters of the model can be calculated.

$$
\begin{aligned}
& \operatorname{Re}\left[G\left(j \omega_{u}, T, \phi\right)\right]=-1 / k_{u} \\
& \operatorname{Im}\left[G\left(j \omega_{u}, T, \phi\right)\right]=0 .
\end{aligned}
$$

As was shown in section two, parameters $\phi$ and $T$ determine the dynamic features of the system. Therefore, it is advantageous to assess these two parameters on the basis of the same identification method. For this reason it is suggested that $T$ be assessed together with $\phi$ on the basis of the relay feedback test. Simplifying (7) the following expressions are obtained

$$
\begin{aligned}
\phi & =\frac{\pi-\arccos \left\{K k_{u} \cos \left(\omega_{u} \tau\right)\right\}}{\omega_{u}} \\
T & =\frac{\sin \left(\omega_{u} \phi\right)-\tan \left(\omega_{u} \tau\right) \cos \left(\omega_{u} \phi\right)}{\omega_{u}} .
\end{aligned}
$$

Identification of the parameters of model (5) is more difficult, because it contains two additional parameters. The possible solution of the identification is to find more points of the frequency response and solve the set of equations numerically, by analogy with (7).

\section{Application to internal model control}

As has been shown in [5] and [6] the conventional internal model control (IMC) design of the controller (Morari, Zafiriou [7]) can be extended to a broad class of time delay systems. With respect to preceding parts of this paper, first order models with time delays (2) and (5) are able to describe a large class of systems. This is the main reason why these models are suitable for application to IMC. Controller $R(s)$ for model (2) is according to the IMC design

$$
R(s)=\frac{T s+\exp (-s \phi)}{K(F s+1)}
$$

where $T, \phi$ and $K$ are parameters of the model. The filter $1 /(F s+1)$, where $F$ is a time constant, ensures the feasibility of the controller. The inner loop with controller $R$ and model of the system $G$ can be described by the transfer function

$$
C(s)=\frac{R(s)}{1-R(s) G(s)}=\frac{T s+\exp (-s \phi)}{K(F s+1-\exp (-s \tau))} .
$$

The control feedback arrangement with controller $C$ acquires a conventional structure. If model (5) is used, the analogous controller results

$$
C(s)=\frac{T s+\exp (-s \phi)}{K[P s+\exp (-s \psi)](F s+1-\exp (-s \tau))} .
$$

Applying controller (11) to model (2) or controller (12) to model (5), a feedback loop of quite simple and favourable dynamics is obtained.

$$
G_{w y}(s)=\frac{C(s) G(s)}{1-C(s) G(s)}=\frac{\exp (-s \tau)}{F s+1} .
$$

If the real system properties agree with models (2) or (5) the control loop is not only always stable, but moreover its transients are without overshoot, given by the only pole $s=-1 / F$. The IMC controller compensates most of the undesirable effects of delays in the system response. In spite of encountering result (13), it should be noted that an exact agreement between the real system and its model (2) or (5) cannot be achieved. For this case, parameter $F$ affects not only the speed but also the robustness of the closed loop.

\section{Example 1 - ball levitation}

An application of the designed control method is demonstrated on a simple laboratory system - ball levitation (see Figure 5). The actuator of the system is a pump. The pump supplies a jet with an appropriate amount of water, so that the ball, which is raised by the water flow, is maintained in the desired horizontal position. The position is sensed by an ultrasound sensor. The controller (11) generates the actuating signal $u$ assigning the pump performance - the amount of water drawn through the jet.

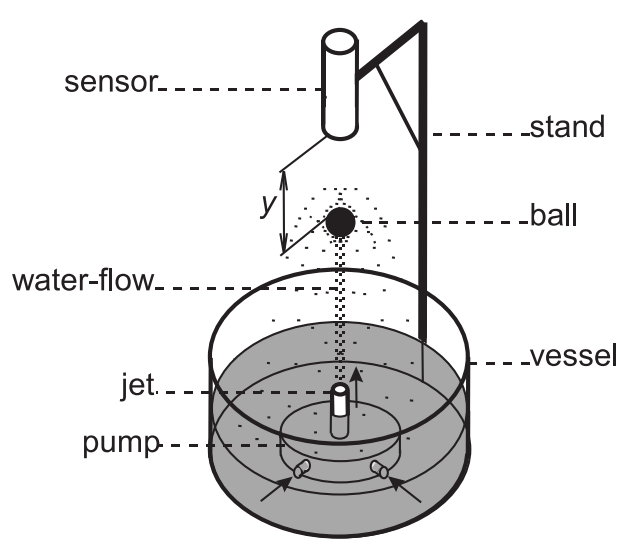

Fig. 5: Laboratory system - ball levitation

Steady state gain $K=62.5$ and input time delay $\tau=0.7 \mathrm{~s}$ were estimated from the step response of the system that is shown in Figure 6. The relay feedback experiment was performed and the following ultimate parameters were found: $\omega_{u}=3.5 \mathrm{~s}^{-1}, k_{u}=0.02$. Parameters $T=0.31 \mathrm{~s}$ and $\phi=0.08 \mathrm{~s}$ were calculated from equations (8) and (9). The simulated step response with assessed parameters is shown in Figure 6. It is apparent that model (2) with identified parameters describes the system dynamics very well.

Because of the higher value of the ratio $\tau / T$ and because of the presence of a distinct noise, a rather higher value of the time constant of the filter $F=1 \mathrm{~s}$ was chosen. The change of desired position of the ball from $w=70 \mathrm{~mm}$ to $w=120 \mathrm{~mm}$, shown in Figure 7, shows a good control response. 


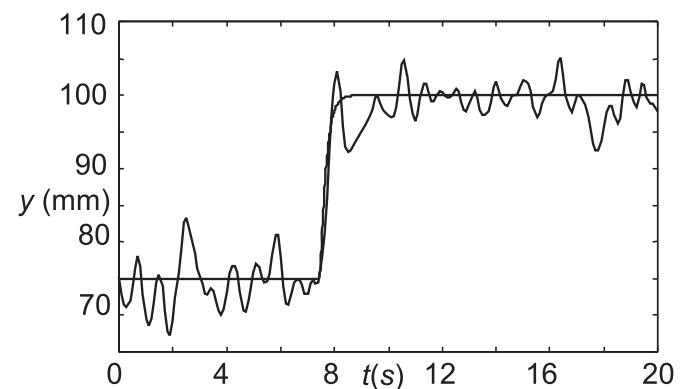

Fig. 6: Step responses of the system and its model

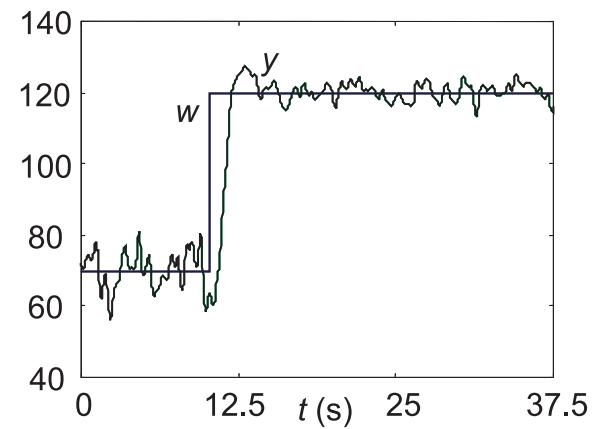

Fig. 7: Set-point response of the closed loop

\section{Example 2 - pitch attitude controller}

The substitution of the higher order model by model (5) and its implementation to IMC is illustrated through application to the pitch attitude controller of an aircraft. The transfer function (14) (Etkin and Reid [8]) describes the dynamic relationship between the rudder angle $\delta_{\mathrm{e}}$ and the pitch angle $\theta$ (see Figure 8 ). Details of the investigated plane, a Boeing

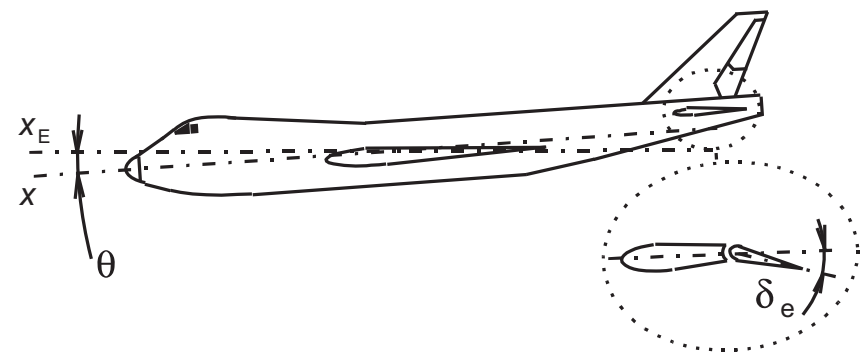

Fig. 8: Definition of pitch angle $\theta$ and rudder angle $\delta_{\mathrm{e}}$ in the motion of the plane

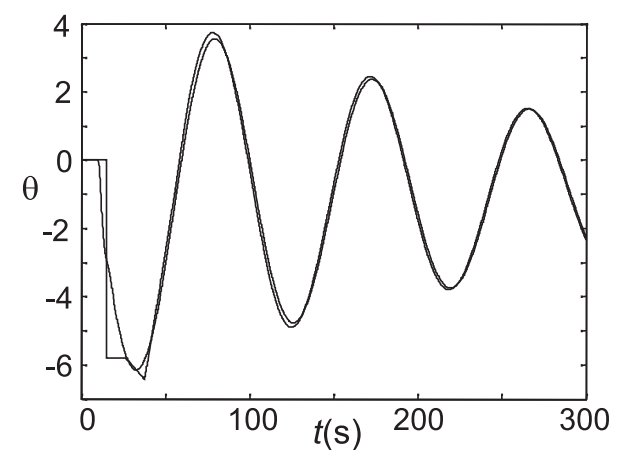

Fig. 9: Step responses of model (14) and its substitution (5)
$747-100$, as well as the methodology used to obtain model (14) can be found in the mentioned literature. The pitch angle is defined as the angle between the plane axes $x$ and the earth fixed axis $x_{\mathrm{E}}$. This angle is readily available from either the real horizon (pilot) or the vertical gyro (auto-pilot).

It should be noted that linear model expressed by the transfer function

$$
G_{\theta, \delta_{e}}(s)=\frac{N(s)}{M(s)}=\frac{-\left(b_{2} s^{2}+b_{1} s+b_{0}\right)}{s^{4}+a_{3} s^{3}+a_{2} s^{2}+a_{1} s+a_{0}}
$$

with parameters $b_{0}=0.003873, b_{1}=0.3545, b_{2}=1.158$, $a_{0}=4.19588 \cdot 10^{-3}, \quad a_{1}=9.463025 \cdot 10^{-3}, \quad a_{2}=0.935494$, $a_{3}=0.750468$ is supposed truly to describe the dynamic relation between $\delta_{\mathrm{e}}$ and $\theta$ only for small departures from the reference position. Let us turn our attention to an investigation of the dynamic properties of model (14). The system has two zeros and four poles. Zeros $p_{1}=-0.0113 \mathrm{~s}^{-1}$ and $p_{2}=-0.2948 \mathrm{~s}^{-1}$ were found directly as two solutions of the equation $N(s)=0$, while the system poles $s_{1,2}=-0.0034 \pm \mathrm{j} 0.0650 \mathrm{~s}^{-1}$ and $s_{3,4}=-0.37 \pm \mathrm{j} 0.8875 \mathrm{~s}^{-1}$ result from numeric calculation of the equation $\mathrm{M}(\mathrm{s})=0$.

The numerator of the alternative model (5), namely parameters $P$ and $\psi$, can be assigned on the basis of found zeros $p_{1}$ and $p_{2}$ from the equation $P s+\exp (-s \psi)=0$. The same distribution of dominant zeros appears if $P=101 \mathrm{~s}$ and $\psi=11.51 \mathrm{~s}$. The steady state gain $K=-0.92305$ is obtained from (14) if $s=0 \mathrm{~s}$. If the dominant couple of the model (14) $s_{\mathrm{F} 1,2}=-0.0034 \pm \mathrm{j} 0.0650 \mathrm{~s}^{-1}$ is prescribed for model (5), the parameters $T=16.64 \mathrm{~s}$ and $\phi=23.4 \mathrm{~s}$ result. Another couple $s_{\mathrm{F} 3,4}=-0.0734 \pm \mathrm{j} 0.3260 \mathrm{~s}^{-1}$ appears near to the imaginary axis. This couple is closer to the origin of the complex domain than couple $s_{3,4}$ of model (14). This causes a certain phase shift between the responses of the models. Better agreement of model (14) and model (5) is achieved if parameters $T=16.1 \mathrm{~s}$ and $\phi=22.61 \mathrm{~s}$ are used. Model (5) then has dominant poles $s_{\mathrm{F} 1,2}=-0.0035 \pm \mathrm{j} 0.0671 \mathrm{~s}^{-1}$ and $s_{\mathrm{F} 3,4}=-0.0758 \pm \mathrm{j} 0.3371 \mathrm{~s}^{-1}$. By means of the last parameter $\tau=5 \mathrm{~s}$ the phase shift is completely removed. Step responses and Bode diagrams of model (14) and its approximation (5) are shown in Figure 9 and Figure 10, respectively. Apparent discrepancies in the beginning of the step responses are caused by the same order of the numerator and the denominator of model (5). This discontinuity is a feature of model (5) and cannot be removed by the choice of different parameters. On the other hand, the step responses are nearly overlapping since they reach their first minimum. The Bode diagrams in Figure 10 show excellent agreement for frequencies

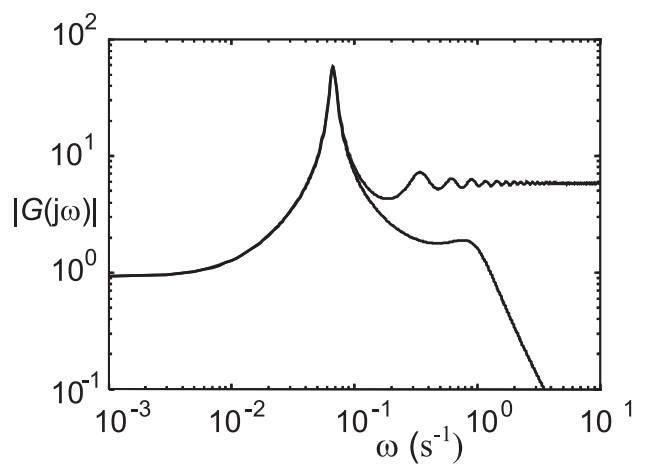

Fig. 10: Bode diagrams of model (14) and its substitution (5) 
$\omega<10^{-1} \mathrm{~s}^{-1}$. Model (5) can be considered as a good approximation of model (14) for lower frequencies. The distinctive discrepancies of the responses after they reach the mentioned boundary value of the frequency indicate that model (5) cannot be used for approximation of model (14) in the higher frequency range. A different set of parameters of model (5) must be used if substitution of model (14) in the higher frequency range is needed.

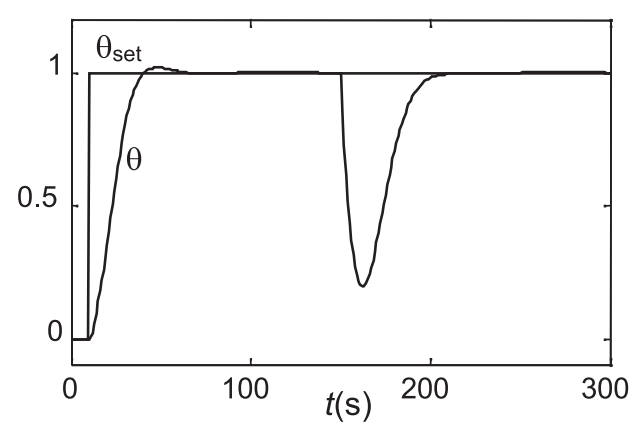

Fig. 11: Set point response and disturbance rejection

The assessed parameters of model (5) were used for controller (12). Model (14) was used as a controlled system in the closed loop. The optional parameter of the controller $F=8 \mathrm{~s}$ ensures good control responses, which are shown in Figure 11, as well as sufficient robustness of the closed loop.

\section{Conclusions}

The presented first order model with input and state delay or with another delay in the model input section proves to have the ability to describe systems with delay dynamics. In spite of its first order, it can be used for describing systems conventionally described by a higher order model. The model is appropriate for describing both non-oscillatory and oscillatory systems with arbitrary dead time. The well-known method based on a closed loop with a relay in combination with a step response may be used for assessing the model parameters. With respect to its plain structure, the model is well suited to be applied in the framework of the internal model control scheme (IMC). It should be noted that the good features of the designed control method depend greatly on the agreement of the model and the system dynamics. This agreement is easy to obtain for industrial or laboratory systems with simple and time-invariant dynamics. The presented control of ball levitation is a typical example of such a system. On the other hand, real systems like the presented pitch attitude control of a plane are mostly non-linear with time-variant dynamics. This fact should be taken into account in controller implementation. The problem of system non-linearity can then be solved by means of linearization and by varying set of IMC controller parameters for each operational state of the system. As regards implementation, the control algorithm can be easily implemented on PLC. The authors implemented the presented IMC controller on the PLC produced by the control technology production company TECO Kolín.

\section{Acknowledgement}

This research was supported by the Ministry of Education of the Czech Republic under Project LN00B096, and by the University of Glasgow, Department of Aerospace Engineering.

\section{References}

[1] Smith, O. J. M.: Closer control of loops with dead time. Chem. Eng. Prog., 1957, 53 (5), pp. 217-219

[2] Gorecki, H., Fuksa, P., Grabowski, P., Korytowski, A.: Analysis and Synthesis of Time Delay Systems. PWN-Polish Scientific Publishers, Warszawa, 1989

[3] Marshal, J. E., Gorecki, H., Walton, K., Koritowski, A.: Time-Delay Systems, Stability and Performance criteria with applications. Ellis Horwood Limited, Chichester, 1992

[4] Astrom, K. J., Hagglund, T.: Automatic tuning of simple regulators with specifications on phase and amplitude margins. Automatica 20/1984, pp. 645-651

[5] Zítek, P.: Time Delay Control System Design Using Functional State Models. CTU Reports No. 1/1998, CTU Prague, p. 93

[6] Zítek, P., Hlava, J.: Anisochronic Internal Model Control of Time Delay Systems. Control Engineering Practice 9, No. 5/2001, pp. 501-516

[7] Morari, M., Zafiriou, E.: Robust Process Control. Prentice-Hall, Englewood Clifts, N. J., 1989

[8] Etkin, B., Reid, L. D.: Dynamics of Flight, Stability and Control. John Wiley \& Sons, Inc., 1996

Ing. Tomáš Vyhlídal

phone: +420224353953

e-mail:vyhlidal@student.fsid.cvut.cz

Prof. Ing. Pavel Zítek, DrSc.

phone: +420224352564

e-mail: zitek@fsid.cvut.cz

Institute of Instrumentation and Control Engineering cAk - Centre for Applied Cybernetics

Czech Technical University in Prague

Faculty of Mechanical Engineering

Technická 4, 16607 Praha 6, Czech Republic 